Self-regulating and diameter-selective growth of $\mathrm{GaN}$ nanowires

This article has been downloaded from IOPscience. Please scroll down to see the full text article.

2006 Nanotechnology 17 S332

(http://iopscience.iop.org/0957-4484/17/11/S17)

The Table of Contents and more related content is available

Download details:

IP Address: 140.112.113.225

The article was downloaded on 09/03/2009 at 05:34

Please note that terms and conditions apply. 


\title{
Self-regulating and diameter-selective growth of GaN nanowires
}

\author{
Chin-Kuei Kuo ${ }^{1,6}$, Chih-Wei Hsu ${ }^{2,6}$, Chien-Ting $\mathbf{W u}^{3}$, \\ Zon-Huang Lan $^{3}$, Chung-Yuan Mou ${ }^{1}$, Chia-Chun Chen ${ }^{2}$, \\ Ying-Jay Yang ${ }^{4}$, Li-Chyong Chen ${ }^{5}$ and Kuei-Hsien Chen ${ }^{3,7}$ \\ ${ }^{1}$ Department of Chemistry, National Taiwan University, Taipei 106, Taiwan \\ ${ }^{2}$ Department of Chemistry, National Taiwan Normal University, Taipei 106, Taiwan \\ ${ }^{3}$ Institute of Atomic and Molecular Sciences, Academia Sinica, Taipei 106, Taiwan \\ ${ }^{4}$ Department of Electrical Engineering, National Taiwan University, Taipei 106, Taiwan \\ ${ }^{5}$ Center for Condensed Matter Sciences, National Taiwan University, Taipei 106, Taiwan \\ E-mail: chenkh@pub.iams.sinica.edu.tw
}

Received 13 December 2005

Published 19 May 2006

Online at stacks.iop.org/Nano/17/S332

\begin{abstract}
We report diameter-selective growth of GaN nanowires (NWs) by using mono-dispersed Au nanoparticles (NPs) on a ligand-modified Si substrate. The thiol-terminal silane was found to be effective in producing well-dispersed Au NPs in low density on Si substrates so that the agglomeration of Au NPs during growth could be avoided. The resultant GaN NWs exhibited a narrow diameter distribution and their mean diameter was always larger than, while keeping a deterministic relation with, the size of the Au NPs from which they were grown. A self-regulating steady growth model is proposed to account for the size-control process.
\end{abstract}

\section{Introduction}

Recently, semiconductor NWs $[1,2]$ have attracted considerable attention as building blocks in electronics [3], optoelectronics [4], and sensors for specific molecular detection [5]. They are of fundamental importance for their special quantum behaviour in band gap engineering [6] and transport mechanisms [7]. Among various semiconductor materials, GaN having a wide direct bandgap is a highly promising material in optoelectronic and electronic applications, such as light-emitting $\mathrm{p}-\mathrm{n}$ junctions and lasers, as well as logic circuits [8-11]. It has already been realized that further exploration and exploitation of new physics and novel devices associated with the NWs will require reliable control of size-selectivity processes.

Previously, we reported the preparation of high-quality GaN NWs by the chemical vapour deposition (CVD) method using gallium and ammonia as precursors [12]. It is known that metal NPs can be used to both catalyze the growth and control the size of the NWs through the vapour-liquid-solid (VLS) mechanism. However, the synthesis of NWs with a narrow diameter distribution with a mean value in the strongconfinement regime is still quite challenging.

6 These two authors contributed equally.

7 Author to whom any correspondence should be addressed.
The Au NPs, reported to be effective for growing the NWs of InP [13], Si [14], and GaAs [15], were found to be catalytically inactive for $\mathrm{GaN}$ due to the poor solubility of $\mathrm{N}$ in $\mathrm{Au}$ [16]. Therefore, the 3rd row transition metals, such as $\mathrm{Fe}$ and $\mathrm{Ni}$, have been most commonly used as catalysts in the VLS growth of GaN NWs, because of the better solubility of $\mathrm{Ga}$ and $\mathrm{N}$ in the liquid metals [12, 16-18]. However, the main drawback of using $\mathrm{Fe}$ and $\mathrm{Ni}$ catalysts is the generation of contamination in the GaN NWs and the corresponding unwanted variation in their electronic properties. A recent computational study [19] observes low defect formation energy for $\mathrm{Ni}$ substitution $(1.2 \mathrm{eV})$ in $\mathrm{GaN}$, where $\mathrm{Au}$ substitution gives higher defect formation energy $(\sim 4 \mathrm{eV})$. One can expect, thus, much less diffusion of $\mathrm{Au}$ atoms into the lattice of GaN. This renders $\mathrm{Au}$ a better choice of catalyst for synthesizing GaN NWs from the point of view of product purity. Other advantages of using Au NP are the simplicity in better sizecontrol and the synthesis of NPs having a size below $10 \mathrm{~nm}$. The detailed preparation method for sized-controlled Au NPs can be found elsewhere [20,21].

In this paper, we present the result of the size-control of GaN NWs using mono-dispersed Au NPs on Si substrate with a ligand modifier, which was used to increase the affinity between $\mathrm{Au}$ and $\mathrm{Si}$ while avoiding the aggregation of 


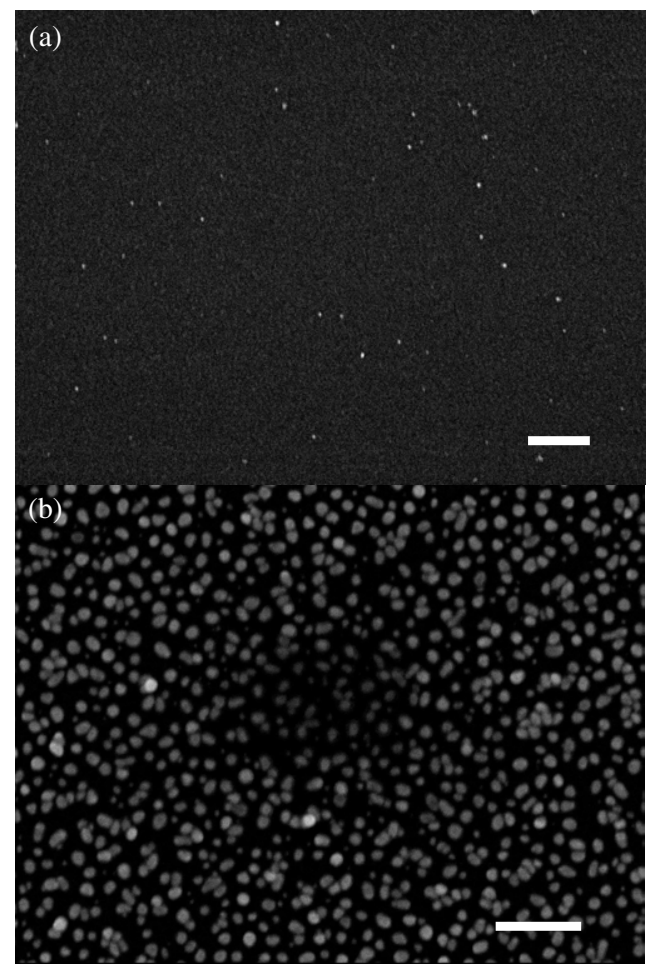

Figure 1. The SEM images of Au NPs on (a) thiol-terminated (MPTMS) Si substrate and (b) amine-terminated (APTMS) Si substrate. $12 \mathrm{~nm}$ Au NPs were used in both cases to clarify the difference in the number density of Au NPs. The white scale bars correspond to $100 \mathrm{~nm}$.

Au NPs during the CVD process. High-resolution transmission electron microscopy (HRTEM) was employed to investigate the structure of the GaN NW and its interface with the $\mathrm{Au}$ NP. Systematic studies of the relationship between the size of the Au NPs, produced by the citrate reduction method, and the diameter of the resultant GaN NWs were performed and an empirical relationship was established over a range of sizes. Finally, as an attempt to address the size-selective phenomena, a self-regulating growth model is proposed.

\section{Experimental details}

$\mathrm{Si}$ wafers, used as the substrate for the growth of GaN NWs, were first rinsed in a buffered HF solution and then immersed in SCI solution for further cleansing and enhancing the surface $\mathrm{Si}-\mathrm{OH}$ coverage to be ready for surface functionalization. The $\mathrm{Si}$ surface was modified by thiol-terminal 3-mercaptopropyltrimethoxysilane (MPTMS) or amine-terminal 2-aminopropyltrimethoxysilane (APTMS). The modified Si substrate thus possesses higher affinity toward $\mathrm{Au}$ NPs due to the strong binding interaction between gold and the ligand. Modified substrates were then immersed in the $\mathrm{Au}$ NP solution, which were prepared by the citrate reduction method [22]. More specifically, we chose the citrate reduction of $\mathrm{HAuCl}_{4}$ in water. The ratio between the reducing/stabilizing agents and $\mathrm{Au}(\mathrm{III})$ (the tri-sodium citrate-to-gold ratio) was varied to control the size of Au NPs from $3 \mathrm{~nm}$ to $100 \mathrm{~nm}$. Four $\mathrm{Au}$ NP samples of different average diameters $(3.2 \pm 0.8 \mathrm{~nm}$,

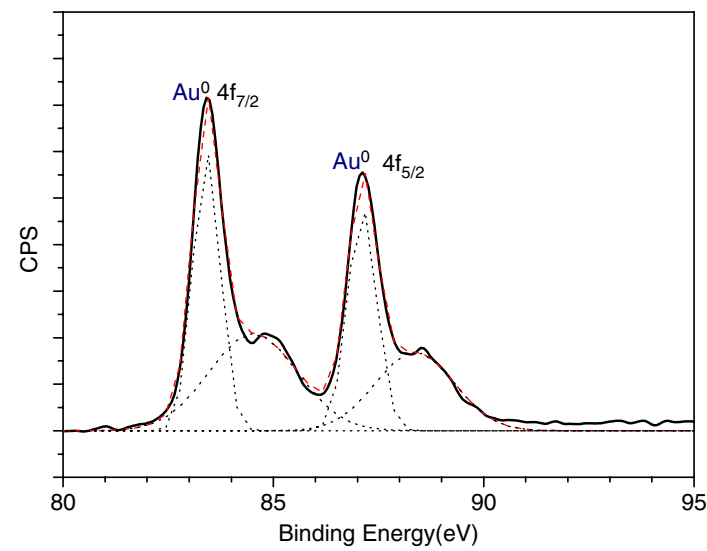

Figure 2. XPS result of MPTMS-modified Si substrates after being immersed in Au NP solution.

(This figure is in colour only in the electronic version)

$7.2 \pm 1.0 \mathrm{~nm}, 12.0 \pm 2.0 \mathrm{~nm}$, and $20.4 \pm 3.4 \mathrm{~nm}$ ) were prepared and examined using the UV-visible spectrum and HR-TEM.

Finally, GaN NWs were grown on the mono-dispersed $\mathrm{Au}$ NP-deposited substrates by using molten gallium as a source material and $\mathrm{NH}_{3}$ as a reactant gas in a quartz tube furnace. The quartz tube was de-gassed under vacuum and purged with $\mathrm{NH}_{3}$. The temperature of the furnace was increased to $920^{\circ} \mathrm{C}$ from room temperature at a rate of $50{ }^{\circ} \mathrm{C} \mathrm{min}^{-1}$ and kept at $920^{\circ} \mathrm{C}$ for $3 \mathrm{~h}$ under a constant flow $(10 \mathrm{sccm})$ of $\mathrm{NH}_{3}$. At this temperature, the organic groups on the Au surface were destroyed and catalytic growth of $\mathrm{GaN}$ could then proceed on the bare surface of gold NPs. After the furnace was cooled to room temperature, white-blue material was found on the surface of the substrate.

X-ray photoelectron microscopy (XPS, VG Scientific, ESCALAB 250) was performed to characterize the surface condition before the growth of NWs. The morphologies and crystal structures of the resulting materials were characterized using field-emission scanning electron microscopy (FESEM, JEOL, JSM-6700F) and x-ray powder diffraction (XRD, Toshiba, A-40-Cu). Further structural and elemental analyses on single NW were performed using high-resolution transmission electron microscopy (HR-TEM, JEOL-400 EX at $400 \mathrm{kV}$ ), selected-area electron diffraction (SAED), and energy-dispersive $\mathrm{x}$-ray spectroscopy (EDX).

\section{Results and discussion}

Comparing the surface of the untreated and different ligandmodified Si substrates after immersion in the Au NP solution, we found that the MPTMS-modified substrate has a lower density of Au NPs and less aggregation of Au NPs compared with the unmodified substrate or APTMS-modified substrate. On the MPTMS-modified surface, the Au NPs have been observed to maintain their individuality (figure 1). The lower density could be due to the repulsive force resulting from the partial charges on the Au particles. We have also measured the XPS spectrum for the MPTMS-supported Au NPs. The presence of the two main peaks (figure 2) at $83.5 \mathrm{eV}$ and $87.1 \mathrm{eV}$ represents the doublet for $\mathrm{Au}^{0} 4 \mathrm{f}_{7 / 2}$ and $4 \mathrm{f}_{5 / 2}$ [23]. The energy shifts of sub-bands at $84.9 \mathrm{eV}$ and $88.5 \mathrm{eV}$ are 

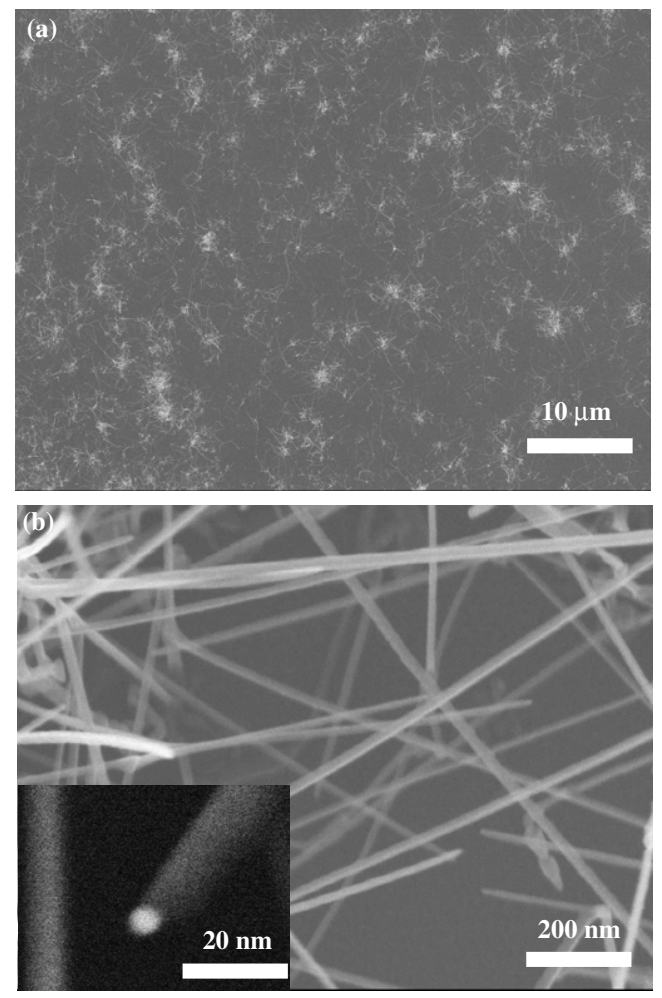

Figure 3. (a) Low-magnification SEM image of GaN NWs on the substrate after the growth process. (b) High-resolution SEM images of GaN NWs grown by using $3.2 \pm 0.8 \mathrm{~nm}$ Au NPs as catalysts. (b) Inset: back-scattered electron image of a $\mathrm{GaN}$ nanowire.

consistent with that reported by McNeillie et al and can be ascribed to the $\mathrm{Au}^{\mathrm{I}}$ in an $\mathrm{Au}-\mathrm{octanethiol} \mathrm{complex} \mathrm{[24].} \mathrm{Thus,}$ $\mathrm{Au}$ NPs were indeed attached to the substrate by the formation of chemical bonding with the sulfur atoms at the end of the modified group. On the other hand, the Au NPs on APTMS are much denser and have been aggregated. At high temperature during the CVD process, the Au NPs would further sinter to an even bigger size. Since a lower number density and lesser aggregation of Au NPs on the substrate are desirable for better controllability of the size-selective growth of GaN NWs, thus, hereafter, we only report the results using MPTMS as the anchoring agent for growth substrate modification.

After the GaN growth process, a thin layer white-blue in colour was observed on the surface of the substrate. A low-magnification SEM image (figure 3(a)) reveals uniform coverage of our resulting materials. High-magnification SEM images of GaN NWs (figure 3(b)) show that GaN NWs are straight in morphology and $\sim 2-7 \mu \mathrm{m}$ in length. A catalytic bead with higher atomic contrast can be observed at the end of the NW from the back-scattered electron image (figure 3(b), inset). High-intensity precision x-ray diffraction analysis of these samples (spectra has not given here) revealed a hexagonal wurtzite structure with lattice constants of $a=3.182 \AA$ and $c=5.178 \AA$. The HR-TEM image (figure 4) further reveals the structural details of the GaN NWs. The SAED pattern (figure 4, inset) can be indexed to the hexagonal GaN crystal along the [001] direction. The corresponding latticeresolved image (figure 4) shows a single-crystalline structure of the individual NW. The spacing of $\sim 0.272 \mathrm{~nm}$ between

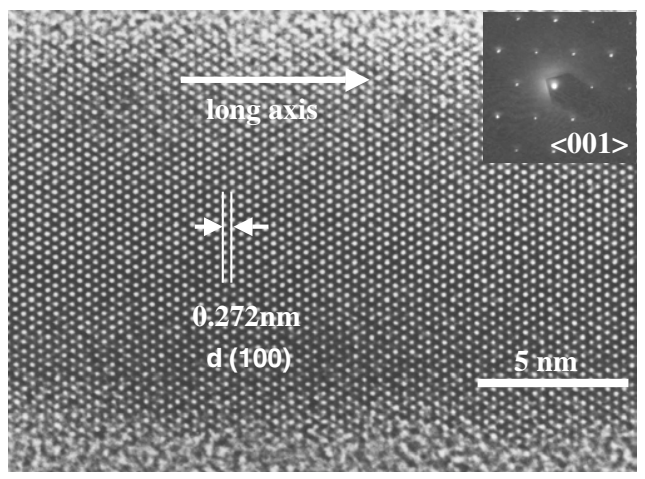

Figure 4. Typical HR-TEM image and its corresponding SAED of a GaN NW. The direction of the long axis is parallel to the [100], and the zone axis is [001]. The diameter of this NW is $\sim 13 \mathrm{~nm}$. The spacing of $0.272 \mathrm{~nm}$ between arrowheads corresponds to the distance between two (100) planes.

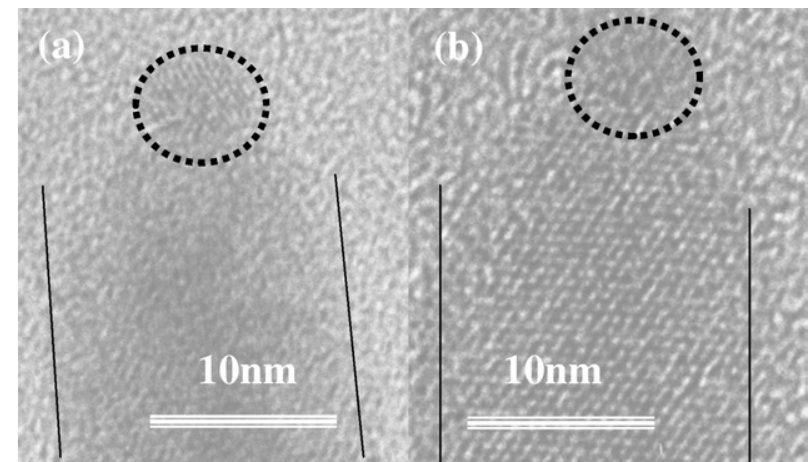

Figure 5. HR-TEM images of two GaN NWs having diameters of (a) $15.8 \mathrm{~nm}$ and (b) $16.8 \mathrm{~nm}$; the dashed circles represent the corresponding catalyst seeds with diameters of (a) 6.8 and (b) $7.1 \mathrm{~nm}$.

the arrowheads corresponds to the distance between two (100) planes, which is in good agreement with that derived from the XRD and SAED results. In our TEM analysis, the growth direction of GaN NWs is always parallel to the (100) planes and no other growth direction is found. It indicates that the GaN NWs are much more phase-pure than our previous results using other transition metals as catalysts [12].

Figure 5 (typical TEM images of the tip of two NWs) reveals that the diameters of two wires are $15.8 \mathrm{~nm}$ and $16.8 \mathrm{~nm}$ and those of the corresponding catalyst seeds (figure 5, the dashed circles) are around $6.8 \mathrm{~nm}$ and $7.1 \mathrm{~nm}$, respectively. Note that the size of the catalyst at the tip of the NW is similar to that before the growth process. A careful study of a sample of a number of NWs has confirmed that the size of the Au catalysts (NPs) at the tips of the NWs remained within the sizerange of the starting Au NPs. It suggests that there was a little coagulation of the Au NPs during the CVD process. Statistical analyses of TEM micrographs further reveals that the diameter of the GaN NW depends on the Au NP size and the diameter distribution of the NWs being considerably narrower compared with those reported in the literature $[25,26]$. A representative diameter distribution for the GaN NWs grown from Au NPs of $7.2 \pm 1.0 \mathrm{~nm}$ is shown in figure 6. By using Au NPs of controlled diameters at 3.2 $\pm 0.8,7.2 \pm 1.0,12 \pm 2.0$, and 


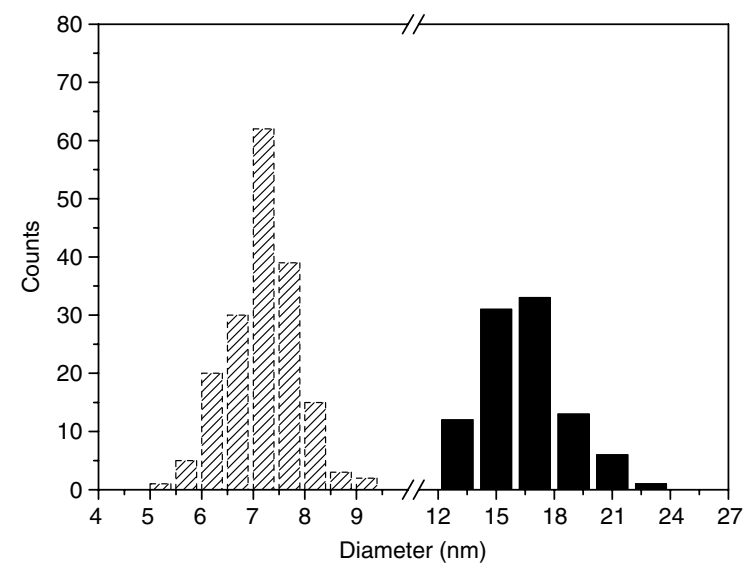

Figure 6. Representative diameter distribution for the GaN NWs of $16 \pm 3.0 \mathrm{~nm}$ (black bars) grown from Au NPs of $7.2 \pm 1.0 \mathrm{~nm}$ (hatched bars). The result of the diameter distribution was estimated from TEM images.

Table 1. Statistical table. (This table shows the mean diameter of the starting Au NPs and the corresponding GaN NWs for four sets of samples. (Mean value \pm standard deviation).)

\begin{tabular}{cl}
\hline Au NPs $(n m)$ & GaN NWs (nm) \\
\hline $3.2 \pm 0.8$ & $10.8 \pm 2.3$ \\
$7.2 \pm 1.0$ & $16.0 \pm 3.0$ \\
$12.0 \pm 2.0$ & $27.2 \pm 3.8$ \\
$20.4 \pm 3.4$ & $51.1 \pm 7.1$ \\
\hline
\end{tabular}

$20.4 \pm 3.4 \mathrm{~nm}$, the mean diameters of the corresponding GaN NWs were found to be $10.8 \pm 2.3,16.0 \pm 3.0,27.2 \pm 3.8$, and $51.1 \pm 7.1 \mathrm{~nm}$, respectively (table 1 ). A linear relationship with a slope of 2.4 has been observed, indicating a stable growth condition in our experiment. The range of the size of GaN NWs is comparable to those reported recently using $\mathrm{Ni}$ thin film [18]. Moreover, in our method, we have a continuous range of mono-dispersed sizes of Au NPs to select. The fact that a clear positive correlation was observed between the size of the catalytic Au NPs and the diameter of the grown NWs also confirms that our process is promising for size-selective growth.

Although Au is not considered to be a good candidate, the successful growth of Au catalyzed GaN NWs has been achieved by several groups $[25,26]$. Experimental results showed that no NW could be obtained on the Si substrates in the absence of Au. This indicated that the presence of Au enhanced the growth of NWs and that the simultaneously anisotropic growth of GaN crystal is unlikely to take place in our system. Investigation by TEM-EDX reveals that only Ga and Au could be detected from the catalytic bead, indicating the possibility of alloy formation. The result is consistent with the previous reports [26], all of which suggested the VLS growth mechanism through binary $\mathrm{Au}-\mathrm{Ga}$ alloy formation. However, neither of the reports could detect the $\mathrm{N}$ signal, and, also, $\mathrm{N}$ has been reported to have a poor solubility in $\mathrm{Au}$ [26]; we suspect that the alloying stage, which is a critical stage of the standard VLS growth mechanism, could not achieve the final ternary phase of $\mathrm{Au}-\mathrm{Ga}-\mathrm{N}$. Recently, Persson et al [27] proposed another growth mechanism, in which NWs can be grown without the alloying state through the vapour-solidsolid (VSS) mechanism. Although our work seems not to follow their VSS mechanism due to the formation of $\mathrm{Au}-\mathrm{Ga}$ alloy, it is worth considering the possibility of the catalytic growth of NWs by a surface diffusion process [27].

In addition, it is also quite intriguing to note that the $\mathrm{Au}$ seed particle is always smaller in diameter than the attached GaN NW (figure 5); at the same time, they are in proportion. For NWs grown by the standard VLS process, the diameter of the seed nanosphere is either larger or equal to the diameter of the growing NWs $[25,26]$. In the present case, the growth of $\mathrm{GaN}$ did not seem to derive directly from the interior of a supersaturated $\mathrm{Au}-\mathrm{Ga}-\mathrm{N}$ alloy seed. More likely, the reaction between $\mathrm{N}$ and $\mathrm{Ga}$ occurred right at the interface between the GaN NW and the seed sphere. Based on our experimental results, we suggest that the growth pathway is likely that $\mathrm{Ga}$ and $\mathrm{Au}$ formed liquid alloy in the first stage, and then this binary $\mathrm{Au}-\mathrm{Ga}$ alloy catalyzed $\mathrm{Ga}$ and $\mathrm{NH}_{3}$ vapour to react and form GaN. Finally, the elongation took place as GaN continued to deposit on the growing tip via $\mathrm{AuGa} / \mathrm{GaN}$ interface, as demonstrated by $\mathrm{Yu}$ et al [26]. It can be rationalized that the $\mathrm{GaN}$ prefers to condense at the existing liquid/solid ( $\mathrm{AuGa} / \mathrm{GaN}$ ) interface, because less energy is required in this step compared to the creation of a new nucleation site. This step might be the controlling kinetic process toward diameter selectivity.

To explain the relation between the Au particle size and the diameter of the attached GaN NW (table 1), we propose a self-regulating growth model in which a NW with a smaller catalytic bead (model I) is preferred over that with a larger catalytic bead (model II). As stated above, we assume that (i) $\mathrm{Ga}$ is activated through the catalyst and the reaction takes place only at the $\mathrm{AuGa} / \mathrm{GaN}$ interface, and (ii) $\mathrm{NH}_{3}$ does not dissolve into the catalyst. Based on (i), Ga can dissolve into an Au NP through the whole surface of the particle. Hence, the in-coming rate of $\mathrm{Ga}$ (abbreviated $R_{\text {in }}$ ) is proportional to the surface area of the $\mathrm{Au} \mathrm{NP}$ and can be expressed as $R_{\mathrm{in}}=\alpha \cdot r_{\mathrm{Au}}^{2}$, where $r_{\mathrm{Au}}$ is the radius of an Au NP, assuming that the NP is a sphere. In such a case, $R_{\text {in }}$ is the same in both models. Regarding the formation of $\mathrm{GaN}$, we first demonstrate model II, in which the Au droplet at the top of GaN NW is larger or equal in diameter to that of the NW (the schematic diagram in figure 7(b)). The reaction can take place due to the diffusion of nitrogen $\left(\mathrm{NH}_{3}\right)$ at the contact circumference between the catalytic bead and NW. This is a diffusion-limited process, since the nitrogen cannot dissolve in gold. Therefore, the forming rate of $\mathrm{GaN}$ is proportional to the radius of NW $\left(r_{\mathrm{NW}}\right)$ and can be expressed as $R_{\mathrm{GaN}, \mathrm{II}}=\delta r_{\mathrm{NW}}$. In model I, in which the Au bead has smaller diameter than the NW diameter, the contact area (at the $\mathrm{Au}-\mathrm{GaN}$ interface) is of negligible value. For simplicity, we can assume that the Au NP stands on the GaN as a full sphere (the schematic diagram in figure 7(a)), which means that $\mathrm{NH}_{3}$ can react directly with the entire top surface (around the $\mathrm{Au}-\mathrm{GaN}$ interface point), neglecting the small contribution of the diffusion process at the point of contact. Therefore, the forming rate of $\mathrm{GaN}$ (abbreviated as $R_{\mathrm{GaN}}$ ) is $R_{\mathrm{GaN}, \mathrm{I}}=\beta r_{\mathrm{NW}}^{2}$.

By plotting $R_{\text {in }}$ and $R_{\mathrm{GaN}}$ versus a reduced length unit $r\left(=r_{\mathrm{NW}} / r_{\mathrm{Au}}\right)$, it can be easily understood that steady growth can occur only in model I (figure 7(a)) while a divergent growth condition is found in model II (figure 7(b)). We should mention 


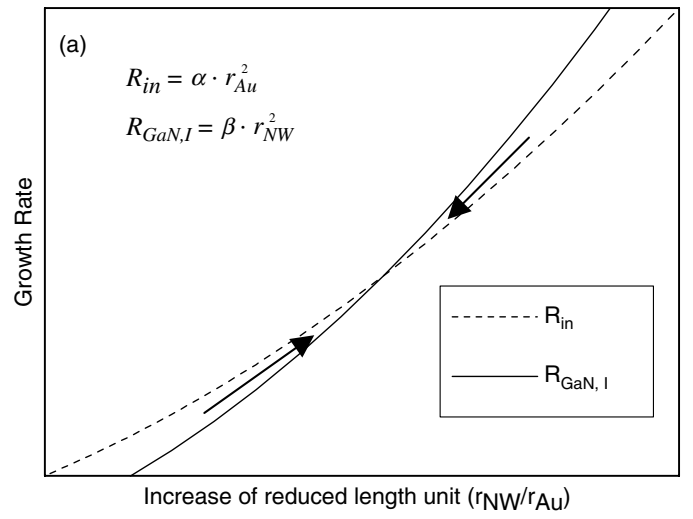

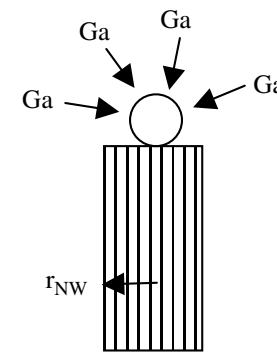

side view

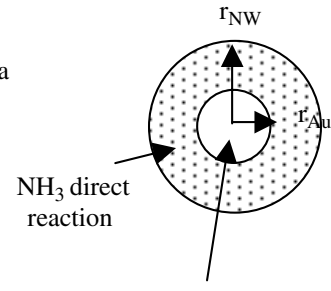

$\mathrm{NH}_{3}$ by diffusion
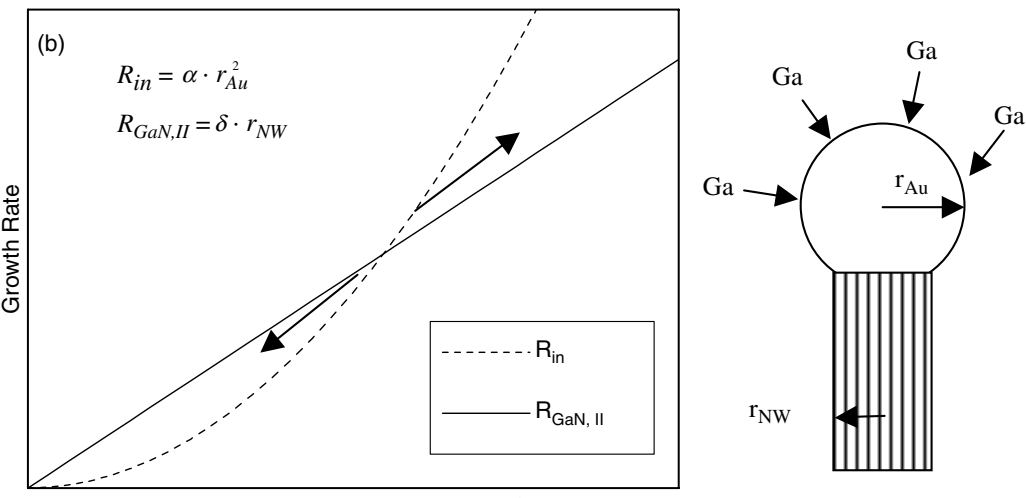

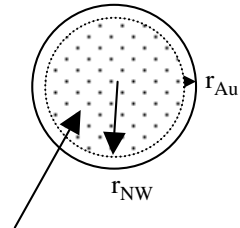

$\mathrm{NH}_{3}$ by diffusion

Figure 7. Plots of Ga in-coming rate $R_{\mathrm{in}}$ and $\mathrm{GaN}$ growth rate $\left(R_{\mathrm{GaN}}\right)$ versus a reduced length unit $\left(r_{\mathrm{NW}} / r_{\mathrm{Au}}\right)$, along with the schematic diagrams for (a) growth model I, where the radius of the catalytic bead $\left(r_{\mathrm{Au}}\right)$ is smaller than that of the NW ( $\left.r_{\mathrm{NW}}\right)$, and (b) growth model II, where $r_{\mathrm{Au}}$ is larger than $r_{\mathrm{NW}}$. Each diagram shows both side and top views of the GaN NW (dotted) with Au NP (white) at the top.

that, in both models, there exists one crossover point, $r_{\mathrm{c}}$, at which $R_{\text {in }}=R_{\mathrm{GaN}}$; any fluctuation from this point could cause either focus back to the steady growth at $r_{\mathrm{c}}$ or divergence to uncontrolled growth. In model II (figure 7(b)), for $r<r_{\mathrm{c}}$, one has $R_{\text {in }}<R_{\mathrm{GaN}, \mathrm{II}}$; the growth of NWs will be under an unstable situation for shrinking, because, if $R_{\mathrm{GaN}, \text { II }}$ is larger than $R_{\text {in }}$, the reaction will be quenched immediately due to the lack of Ga source. For $r>r_{\mathrm{c}}$, one has $R_{\text {in }}>R_{\mathrm{GaN}, \mathrm{II}}$, so that the catalytic bead will grow continuously bigger and bigger and the NWs will be under an unstable situation for growth. In short, the growth will diverge from the equilibrium point $\left(r_{\mathrm{c}}\right)$ if $R_{\text {in }} \neq R_{\mathrm{GaN}, \mathrm{I}}$ in model II. On the other hand, in model I (figure 7(a)), no matter if $r>r_{\mathrm{c}}$ or $r<r_{\mathrm{c}}$ at the initial stage, the growth of NWs converges to $r_{\mathrm{c}}$ (where $R_{\mathrm{in}}=R_{\mathrm{GaN}, \mathrm{I}}$ ). For $r<r_{\mathrm{c}}, R_{\mathrm{in}}>R_{\mathrm{GaN}, \mathrm{I}}$, more Ga will dissolve into the $\mathrm{Au}-\mathrm{Ga}$ alloy bead; excess $\mathrm{Ga}$ will be separated out and will take part in the GaN growth reaction; and $R_{\mathrm{GaN}, \mathrm{I}}$ will increase towards the equilibrium point $\left(r_{\mathrm{c}}\right)$. Beyond $r_{\mathrm{c}}\left(r>r_{\mathrm{c}}\right)$, one has $R_{\mathrm{in}}<R_{\mathrm{GaN}, \mathrm{I}}$ or faster Ga consumption, which will slow down the reaction (or $R_{\mathrm{GaN}, \mathrm{I}}$ ) and the condition will tend to return at $r_{\mathrm{c}}$. Therefore, we can consider that the steady growth model (model I) is preferable for the growth pathway of our GaN NWs. Same self-regulating growth mechanism in model I can also explain the size-selective phenomena. The convergence of growth towards a certain critical point $r_{\mathrm{c}}$ means that there always exists a $r_{\mathrm{NW}}$ for a certain $r_{\mathrm{Au}}$, for a particular growth system. At least we can claim to have a steady growth condition from the evidence that the diameter of our GaN NWs does show a deterministic relation (2.4) to the starting Au NP size. However, we are not able to account for the physical interpretation of the rate constants $\alpha, \beta$ and $\delta$ precisely at this stage, and that is the reason why we are unable to conclude any numerical value for the critical or equilibrium point $r_{\mathrm{c}}$. Further experiments are underway.

\section{Conclusion}

In summary, we have demonstrated that solution-monodispersed Au NPs of distinct sizes on the ligand (MPTPS) modified substrate can be used to catalyze the large-scale, diameter-selective growth of GaN NWs. The narrowest diameter that we can obtain is around $10 \mathrm{~nm}$. An interesting observation has been found that the diameter of the Au NP at the top of GaN NW is always smaller but proportional to the diameter of the corresponding GaN NW. The growth may involve a self-regulating size-control kinetic pathway, somewhat different to the standard VLS mechanism. A possible mechanism for the growth process has been proposed to explain the unique observations.

\section{Acknowledgments}

This work was financially supported by the Ministry of Education and National Science Council, Taiwan. We also acknowledge Dr S Dhara, MSD, IGCAR, Kalpakkam, India for helpful discussions during the preparation of this manuscript. 


\section{References}

[1] Hu J T, Odom T W and Lieber C M 1999 Acc. Chem. Res. 32435

[2] Xia Y, Yang P, Sun Y, Wu Y, Mayers B, Gates B, Yin Y, Kim F and Yan H 2003 Adv. Mater. 15353

[3] Duan X, Huang Y, Cui Y, Wang J and Lieber C M 2001 Nature 40968

[4] Huang H M, Mao S, Feick H, Yan H Q, Wu Y Y, Kind H, Weber E, Russo R and Yang P D 2001 Science 2921897

[5] Cui Y, Wei Q, Park H and Lieber C M 2001 Science 2931289

[6] Ma D D D, Lee C S, Au F C K, Tong S Y and Lee S T 2003 Science 2991874

[7] Zhang Z, Sun X, Dresselhaus M S and Ying J Y 2000 Phys. Rev. B 614850

[8] Fasol G 1996 Science 2721751

[9] Zhong Z, Qian F, Wang D and Lieber C M 2003 Nano Lett. 3343

[10] Johnson J C, Choi H J, Knutsen K P, Schaller R D, Yang P and Saykally R J 2002 Nat. Mater. 1106

[11] Huang Y, Duan X, Cui Y, Lauhon L J, Kim K H and Lieber C M 2001 Science 2941313

[12] Chen C C, Yeh C C, Chen C H, Yu M Y, Liu H L, Wu J J, Chen K H, Chen L C, Peng J Y and Chen Y F $2001 \mathrm{~J}$. Am. Chem. Soc. 1232791

[13] Gudiksen M S and Lieber C M 2000 J. Am. Chem. Soc. 1228801

[14] Gudiksen M S, Wang J and Lieber C M 2001 J. Phys. Chem. B $\mathbf{1 0 5} 4062$

[15] Yu H and Buhro W E 2003 Adv. Mater. 15416

[16] Duan X and Lieber C M 2000 J. Am. Chem. Soc. 122188
[17] Chang K W and Wu J J 2002 J. Phys. Chem. B 1067796

[18] Chen X, Xu J, Wang R M and Yu D 2003 Adv. Mater. 15419

[19] Chisholm J A and Bristowe P D 2001 Comput. Mater. Sci. 2273

[20] Jana N R, Gearheart L and Murphy C J 2001 Langmuir 176782

[21] Handley D A 1989 Colloidal Gold: Principles, Methods, and Applications vol 2, ed M A Hayat (San Diego, CA: Academic) chapter 2

[22] Schmitt J, Mächtle P, Eck D, Möhwald H and Helm C A 1999 Langmuir 153256

[23] Brust M, Walker M, Bethell D, Schiffrin D J and Whyman R 1994 J. Chem. Soc., Chem. Commun. 801

[24] McNeillie A, Brown D H and Smith W E 1980 J. Chem. Soc., Dalton Trans. 767

[25] Chatterjee A, Chattopadhyay S, Hsu C W, Shen C H, Chen L C, Chen C C and Chen K H 2004 J. Mater. Res. 191768

Dhara S, Datta A, Wu C T, Lan Z H, Chen K H, Wang Y L, Chen L C, Hsu C W, Lin H M and Chen C C 2003 Appl. Phys. Lett. 82451

Lee S K, Choi H J, Pauzauskie P, Yang P D, Cho N K, Park H D, Suh E K, Lim K Y and Lee H J 2004 Phys. Status Solidi b 2412775

Han S, Jin W, Tang T, Zhang D, Liu X, Han J and Zhou C 2003 J. Mater. Res. 18245

[26] Cao C, Xiang X and Zhu H 2005 J. Cryst. Growth 273375

Yu Z, Yang Z, Wang S, Jin Y, Liu J G, Gong M and Sun X 2005 Chem. Vapor Depos. 11433

[27] Persson A I, Larsson M W, Stenstrom S, Ohlsson B J, Samuelson L and Wallenberg L R 2004 Nat. Mater. 3677 